\title{
A la poursuite du patrimoine
}

Les lieux de mémoire de l'esclavage dans départements d'outre mer (DOM)

\section{Thierry Nicolas}

\section{OpenEdition}

Journals

\section{Édition électronique}

URL : http://journals.openedition.org/echogeo/11300

DOI : 10.4000/echogeo. 11300

ISSN : 1963-1197

\section{Éditeur}

Pôle de recherche pour l'organisation et la diffusion de l'information géographique (CNRS UMR 8586)

\section{Référence électronique}

Thierry Nicolas, «A la poursuite du patrimoine », EchoGéo [En ligne], Sur le Vif, mis en ligne le 09 juillet 2009, consulté le 19 avril 2019. URL : http://journals.openedition.org/echogeo/11300 ; DOI : 10.4000/ echogeo. 11300

Ce document a été généré automatiquement le 19 avril 2019

\section{(c) (i) (9)}

EchoGéo est mis à disposition selon les termes de la licence Creative Commons Attribution - Pas d'Utilisation Commerciale - Pas de Modification 4.0 International 


\section{A la poursuite du patrimoine}

\section{Les lieux de mémoire de l'esclavage dans départements d'outre mer} (DOM)

\section{Thierry Nicolas}

1 Depuis l'extrême fin du XXe siècle, de nouveaux marqueurs du paysage apparaissent et se multiplient dans les quatre départements d'outre-mer (Guadeloupe, Martinique, Guyane, La Réunion) afin de commémorer l'une des pages les plus sombres du passé de ces territoires : l'esclavage. Aucune de ces «vieilles colonies» devenues parties intégrantes de la Nation en 1946 n'échappe désormais à ce processus d'élaboration de lieux de mémoire ${ }^{1}$. De Rémire-Montjoly à Saint-Paul en passant par Saint-François ou Les Troisilets, les témoins de la résurgence de la mémoire de l'esclavage s'inscrivent dans le quotidien des ultramarins en occupant notamment des emplacements privilégiés tels que les places publiques, les squares, les fronts de mer ou les ronds-points. Toutefois, en dépit de leur empreinte paysagère, ceux-ci peinent à être qualifiés de patrimoniaux comme en témoignent d'une part leur absence dans les ouvrages spécialisés sur le patrimoine et les guides touristiques présentant les départements d'outre-mer, et d'autre part, la faible place et le peu d'intérêt que leur accordent les instances culturelles lors, par exemple, des journées du patrimoine. Cet article se propose par conséquent de porter un éclairage sur la difficile intégration des lieux de mémoire de l'esclavage au patrimoine. Il s'agit en effet de mieux appréhender, à partir des exemples que l'on retrouve dans les départements d'outre-mer insulaires, la complexité du processus de patrimonialisation de lieux de mémoire aussi singuliers.

\section{Des lieux de mémoire « anciens » comme lieus d'histoire}

2 Si allier les lieux de mémoire de l'esclavage au patrimoine ne semble pas aller de soi, en revanche pour les lieux de mémoire qui ont été élaborés avant la fin des années 1990, le lien est plus évident. Cette différence ne tient ni à un simple recul temporel ni à une quelconque qualité esthétique de ces marqueurs mémoriels, mais est plutôt liée à la place qu'occupe la discipline historique dans leur logique d'élaboration. Il s'avère en réalité que 
les lieux de mémoire « anciens » qui aujourd'hui encore jalonnent le paysage ultramarin sont davantage marqués par le sceau de l'histoire. A la différence des lieux de mémoire de l'esclavage, ils apparaissent comme le résultat d'un exercice réglé peu tributaire des enjeux communautaires et identitaires qui se cachent derrière la mémoire. Pour s'en convaincre, il suffit de recenser les principaux lieux de mémoire aujourd'hui visibles qui témoignent de cette époque et remarquer qu'ils ont comme point commun de légitimer un savoir institué et "laïcisant». Ils peuvent d'ailleurs être divisés en deux sousensembles. D'un côté, on retrouve les lieux de mémoire célébrant les figures « historiques » et de l'autre, les cénotaphes rappelant le rôle joué par les Ultramarins lors des deux guerres mondiales.

\section{Des figures de la mémoire aux figures de l'histoire}

3 Il est possible de regrouper dans un premier sous-ensemble une série de lieux de mémoire dont la logique d'édification répondait à la volonté de valoriser les personnages qui ont marqué «l'Histoire» des ces îles. Parmi ceux-ci se dégagent les figures «métropolitaines » dont l'empreinte a été forte et les personnalités locales qui ont connu un destin hors du commun.

Lorsque l'on évoque les personnalités hexagonales remarquables des départements d'outre-mer le personnage qui revient le plus souvent est Victor Schœlcher surtout aux Antilles et en Guyane. Ce symbole de l'abolitionnisme est généralement représenté sous la forme de bustes et de statues dont la plus connue reste celle le présentant en train d'accompagner un enfant sur le chemin de l'éducation puisqu'on la retrouve à Cayenne (cf. photo 1) et à Fort-de-France. 
Photo 1 - Statue de Victor Schœlcher accompagnant un enfant sur le chemin de l'éducation à Cayenne (Guyane)

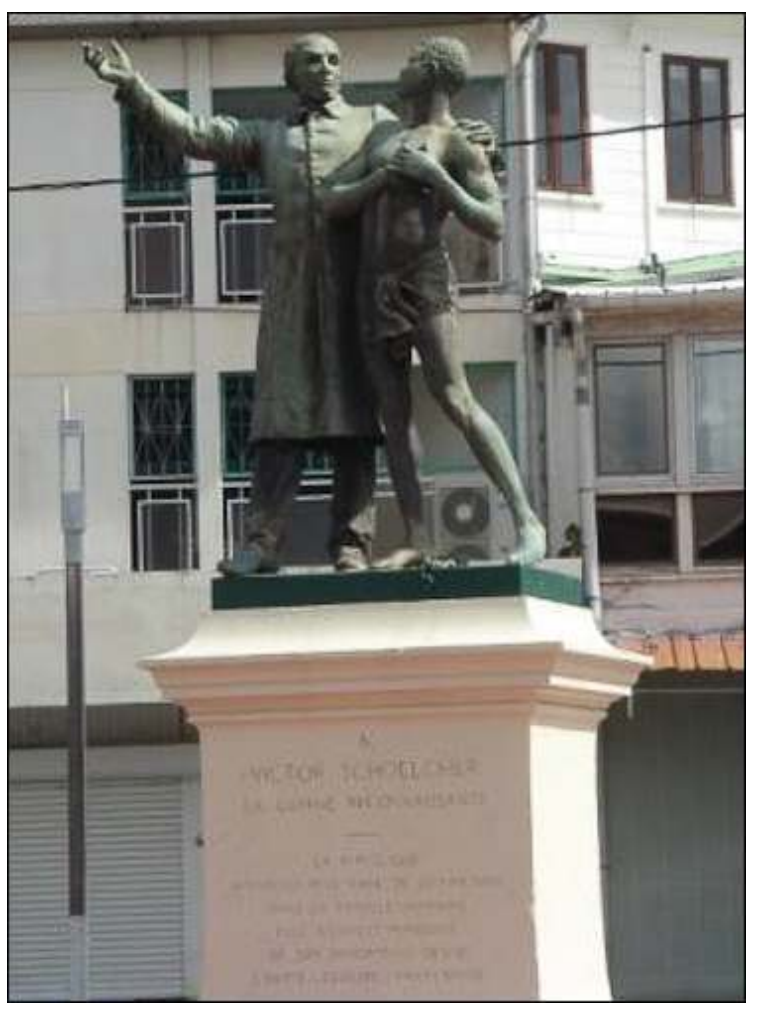

Cliché Isabelle Hidair

5 A côté de ce personnage central, les figures marquantes de la colonisation occupent une place de choix qu'il s'agisse des premiers découvreurs ou des gouverneurs importants. A la Réunion se trouve, faisant face à la Préfecture, la statue de Mahé de Labourdonnais (cf. photo 2) - le gouverneur qui, au XVIII siècle, stimula l'activité économique de l'île Bourbon -, tandis qu'à la Martinique, dominant la Baie des flamands, on peut observer celle de Belain d'Esnambuc à l'origine de la colonisation de l'île (cf. photo 3). 
Photo 2 - Statue de Belain d'Esnambuc face à la Baie des Flamands (Fort-de-France, Martinique)

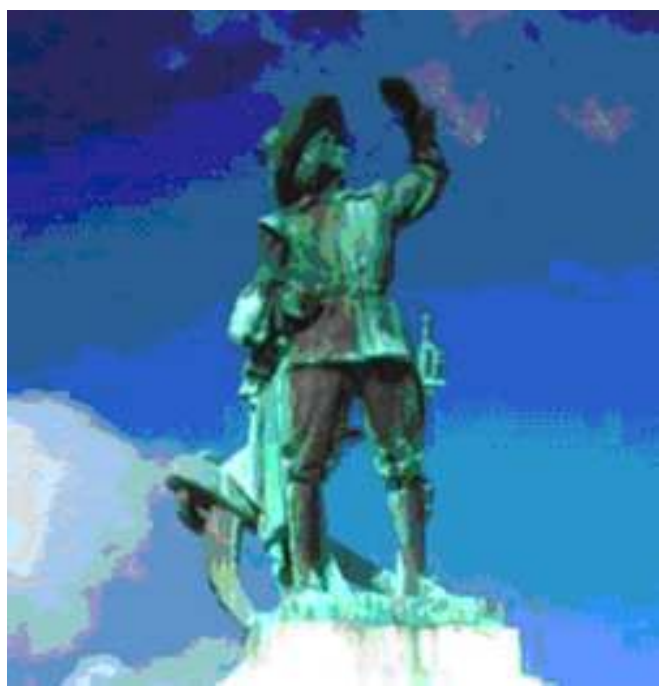

Cliché de l'auteur

Photo 3 - Statue de Mahé de Labourdonnais à Saint-Denis (La Réunion)

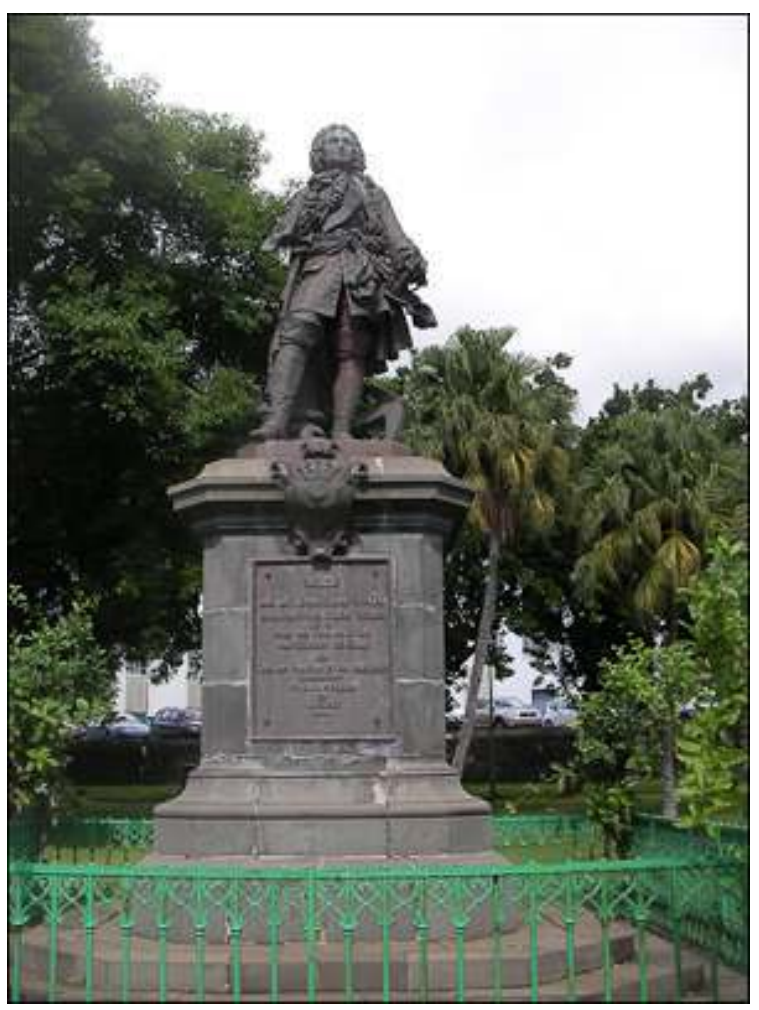

Cliché de l'auteur

6 Concernant les personnalités locales dont le destin sort de l'ordinaire, se distingue l'œuvre montrant sur la place de la Savane à Fort-de-France, Joséphine de Beauharnais², en manteau impérial lors de la cérémonie de couronnement de Napoléon $1^{\mathrm{er}}$. On peut également citer celle représentant 
face au Barachois à Saint-Denis, l'aviateur né à l'île de la Réunion, Roland Garros, qui a laissé son patronyme au fameux tournoi de tennis parisien.

Photo 4 : Statue de Roland Garros au Barachois à Saint-Denis (La Réunion)

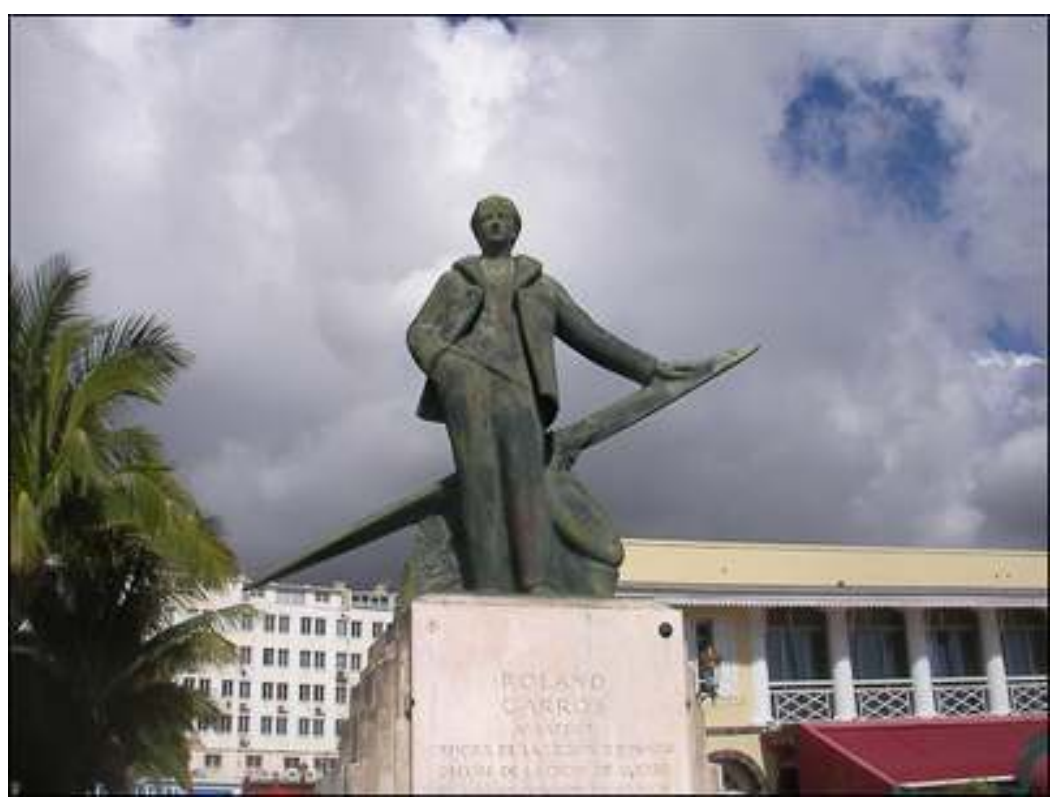

Cliché de l'auteur

\section{Les monuments aux morts comme éléments du récit historique}

7 Dans un second sous-ensemble, il est possible d'intégrer les monuments aux morts construits à la gloire des soldats d'outre-mer ayant donné leur vie à la Nation lors des deux guerres mondiales (1914-18 et 1939-1945). Ces cénotaphes qui jalonnent les communes des quatre DOM ont également acquis une dimension profondément historique dont témoigne l'amen laïc "morts pour la Patrie». Dans la lignée des monuments aux morts des communes de l'Hexagone, ils se présentent le plus souvent sous la forme d'une stèle où apparait une liste des hommes tombés pour la France. Cependant, des formes plus originales se distinguent comme le cénotaphe des Avirons ou celui de Fonds Saint-Denis qui s'individualise par la représentation d'un « poilu » noir. 
Photo 5 : Le monument aux morts des Avirons (La Réunion)

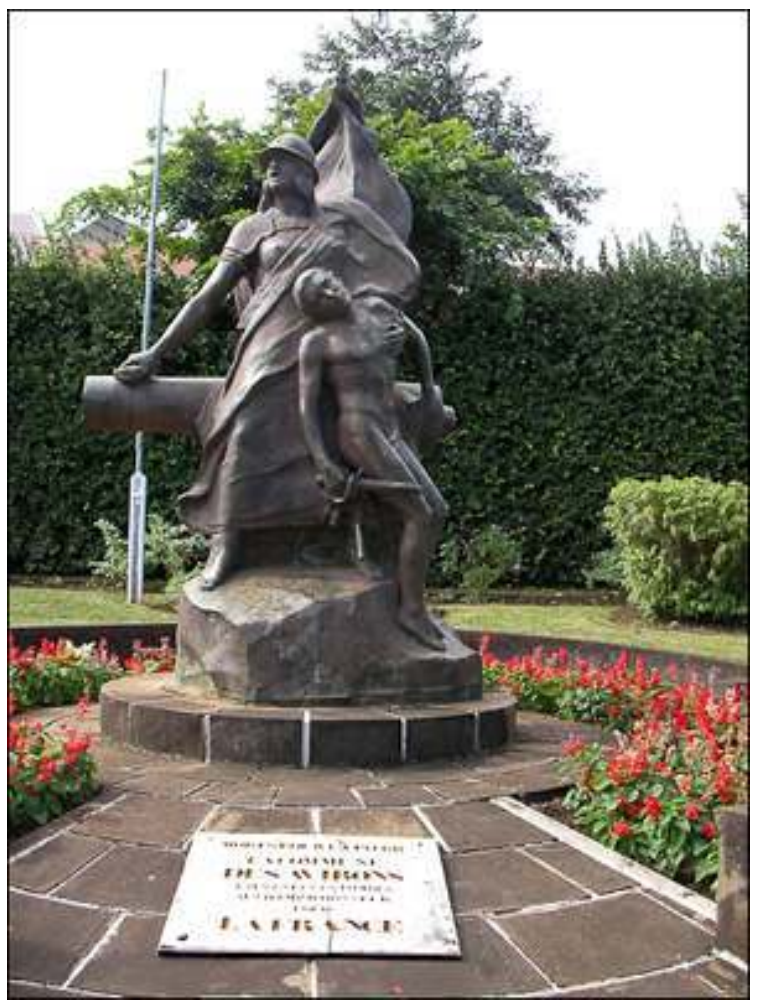

Cliché de l'auteur

Photo 6 : Le monument aux morts de Fonds Saint-Denis (Martinique)

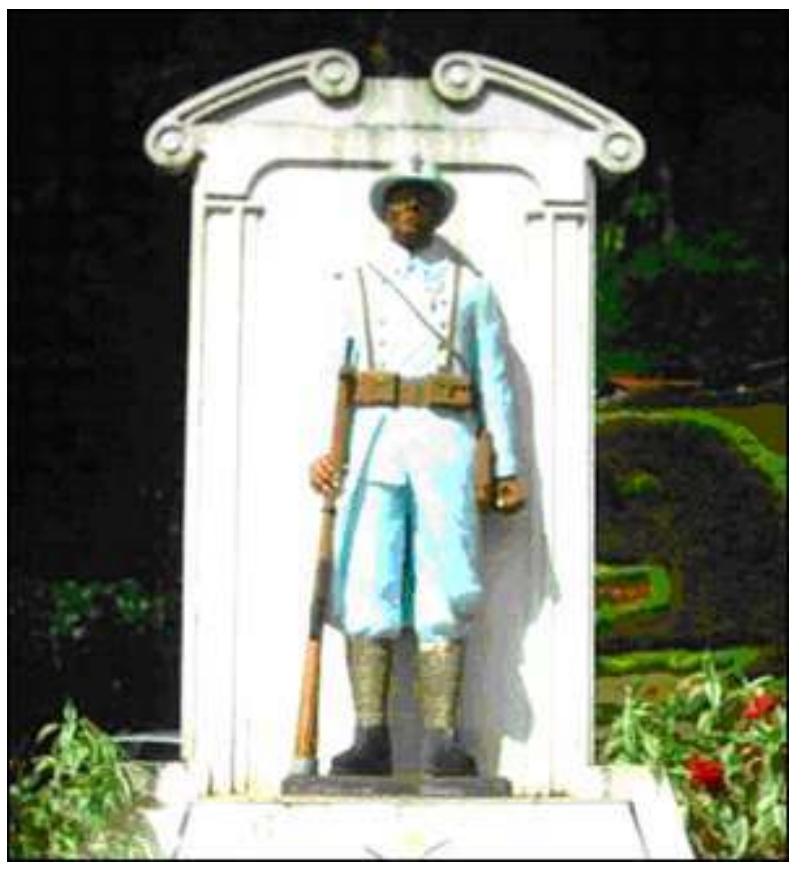

Cliché de l'auteur

8 On remarque qu'en dépit d'évidentes dissemblances entre les cénotaphes et les statues de « grands» personnages, ces lieux de mémoire «anciens» ont comme point commun 
d'être empreints du regard historique. D'ailleurs les mesures de protection dont bénéficient ces monuments qui passent parfois par leur classement font que l'on peut les considérer comme des lieux d'histoire.

\section{Les lieux de mémoire de l'esclavage ou de la mémoire recouvrée}

9 A la différence des lieux de mémoire précédents, la dynamique à l'origine des lieux de mémoire qui ont émergé depuis la fin des années 1990 n'est pas historicisante. Elle est portée par la mémoire qui engage à la fois l'identité des ultramarins et leur sensibilité au passé. L'objectif principal des ultramarins par le biais notamment d'associations, est désormais de faire émerger et revivre le souvenir des esclaves qui a été jusqu'ici négligé. Cette volonté dont l'origine coïncide avec les festivités liées au cent cinquantième anniversaire de l'abolition de l'esclavage rompt ainsi avec près d'un siècle et demi d'oubli ${ }^{3}$ . En effet, ni la commémoration du centenaire de cette seconde abolition en 1948, ni l'octroi de journée de célébration fériée dans chaque DOM en $1983^{4}$, n'ont suscité une dynamique équivalente.

\section{Esclavage, assimilation et séparatisme}

10 Pour comprendre cette situation, il faut se rappeler que les départements d'outre-mer ont connu la politique coloniale de l'assimilation qui traitait « les colonies comme une partie intégrante du territoire»(Girault, 1903) 5 . Destinée à gommer les différences entre la mère-patrie et les colonies, celle-ci s'accompagnait d'un récit historique conforme au roman national. Il n'était pas question de "tolérer que des écoles, des musées, des monuments enseignent ou célèbrent des récits divergents sur le sol même de la nation » (Keslassy, Rosembaum, 2007). L'histoire n'avait pas pour fin de promouvoir un passé qui s'accommodait mal au récit-cadre d'autant que deux éléments venaient conforter cette situation.

11 En premier lieu, le voile posé sur l'esclavage était partagé par toutes les composantes de la société. Comme l'affirment les historiens Jean Poirier et Sudel Fuma « les descendants d'affranchis, eux aussi éprouvaient la même réserve puisqu'il apparaissait honteux d'avoir eu des ascendants en servitude » (Poirier, Fuma, 2004). Il s'agissait en effet d'un héritage si lourd à porter que rares étaient ceux qui se glorifiaient de leur filiation avec des esclaves.

12 En second lieu, évoquer cette époque apparaissait comme une menace au projet d'intégration de ces territoires. Une telle attitude passait pour contraire à la volonté d'assimilation des « vieilles colonies » à la métropole surtout que dans la seconde moitié du XX siècle l'idée de réhabiliter l'esclavage a largement été véhiculée par des mouvements autonomistes et indépendantistes.

13 Ce faisant, les contextes respectifs du centenaire de l'abolition de l'esclavage (1948) célébré au lendemain de la départementalisation - et de la mise en place de journées de célébration (au début des années 1980), en pleine vague d'indépendantisme antillais dur ${ }^{6}$, se prêtaient mal au retour de la question de l'esclavage sur la scène publique. Lors de la préparation, entre 1981 et 1983, des projets de loi de commémoration de l'esclavage et de décret fixant les dates de célébration dans chaque département, cette réserve à l'égard de 
l'esclavage était particulièrement sensible chez certains élus. L'historien Prosper Eve nous rapporte que lors de la consultation du Conseil général de La Réunion en janvier 1982, la majorité d'alors avait proposé au gouvernement d'associer l'abolition de l'esclavage à la départementalisation selon la formulation suivante :

« Projet de loi. Article unique : La commémoration de l'abolition de l'esclavage et de l'érection des quatre vieilles colonies en département français fera l'objet d'une même journée fériée et chômée dans les départements de la Guadeloupe, de la Guyane, de la Martinique et de La Réunion ».

«Projet de décret. Article $1^{\mathrm{er}}$ : Dans les départements d'outre-mer, la date de commémoration de l'abolition de l'esclavage et de l'érection des quatre vieilles colonies en département français est ainsi fixée: La Réunion: 20 décembre.» (Prosper Eve, 2005)

14 En fait, tout se passait comme si le souhait de rouvrir cette page sombre du passé des vieilles colonies renforçait l'insécurité politique et statutaire des quatre départements d'outre-mer.

\section{L'extrême diversité des lieux de mémoire de l'esclavage}

Avec la dissociation progressive du couple liant l'esclavage à la revendication séparatiste depuis les années 1990 et la capacité des descendants d'esclaves à affronter ce passé notamment lors de la commémoration du cent cinquantième anniversaire de son abolition, la mémoire de l'esclavage trouve un contexte propice à son expression. Dans ce cadre, les lieux de mémoire apparaissent véritablement comme les témoins principaux de la réappropriation et de l'acceptation d'un passé difficile. Ceux-ci se présentent aujourd'hui sous des formes extrêmement diverses (mémorial, bustes, statues, fresques, plaques,...) en raison de la variété des thèmes développés. On peut en distinguer cinq qui dominent très nettement :

Une première catégorie de lieux de mémoire rappelle l'origine géographique des esclaves. Leur but principal est de mettre au premier plan les liens qui ont existé avec les deux côtes africaines (orientales et occidentales) et Madagascar. Il en va ainsi par exemple du mémorial de l'Anse Caffard au Diamant, conçu par le plasticien martiniquais Laurent Valère. Réalisé avec du sable de Trinidad, cet ensemble monumental se compose de sculptures disposées en triangle et orientées au cap $110^{\circ}$, en direction du Golfe de Guinée. C'est aussi le cas des statues du front de mer de Saint-Paul réalisées en 2005 par les sculpteurs Rabemananjara et Dolaine Courtis qui interpellent le passant sur le trafic d'esclaves entre Fort Dauphin (Madagascar) et La Réunion. 
Photo 7 - Le mémorial Cap 110 de l'Anse Caffard (Diamant, Martinique)

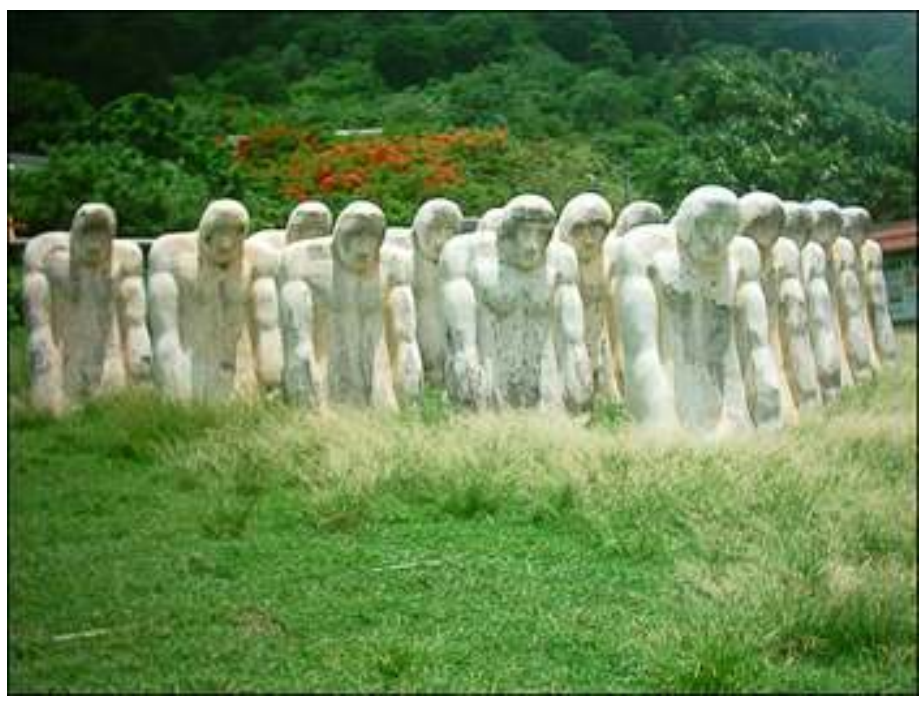

Cliché de l'auteur

Photo 8 - Statues de la route de l'esclavage à Saint-Paul (La Réunion)

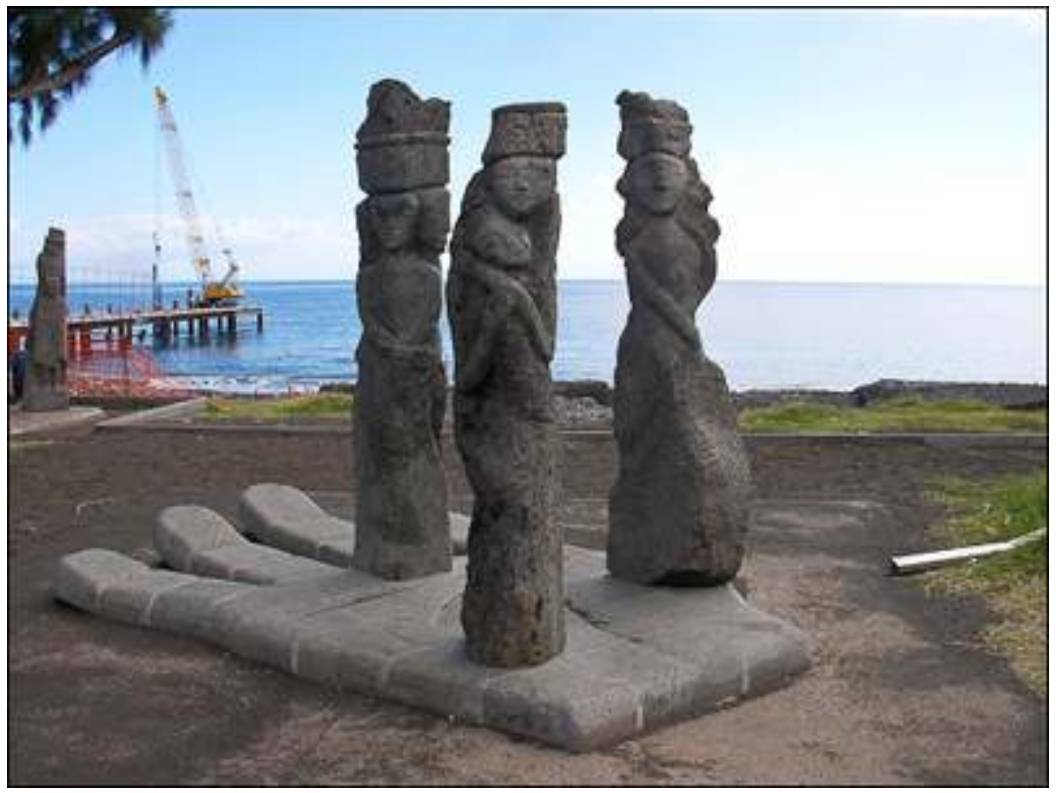

Cliché del'auteur

17 Une seconde catégorie de lieux de mémoire renvoie aux attributs de l'esclave. Il s'agit le plus souvent d'une mise en scène des éléments qui ont marqué l'univers de la condition servile comme les chaînes, les boulets ou les tambours. A Saint-François en Guadeloupe, un mémorial associe ces symboles alors que sur le littoral de Saint-Paul à la Réunion c'est une œuvre monumentale représentant les fers des esclaves ouverts qui domine le front de mer. 


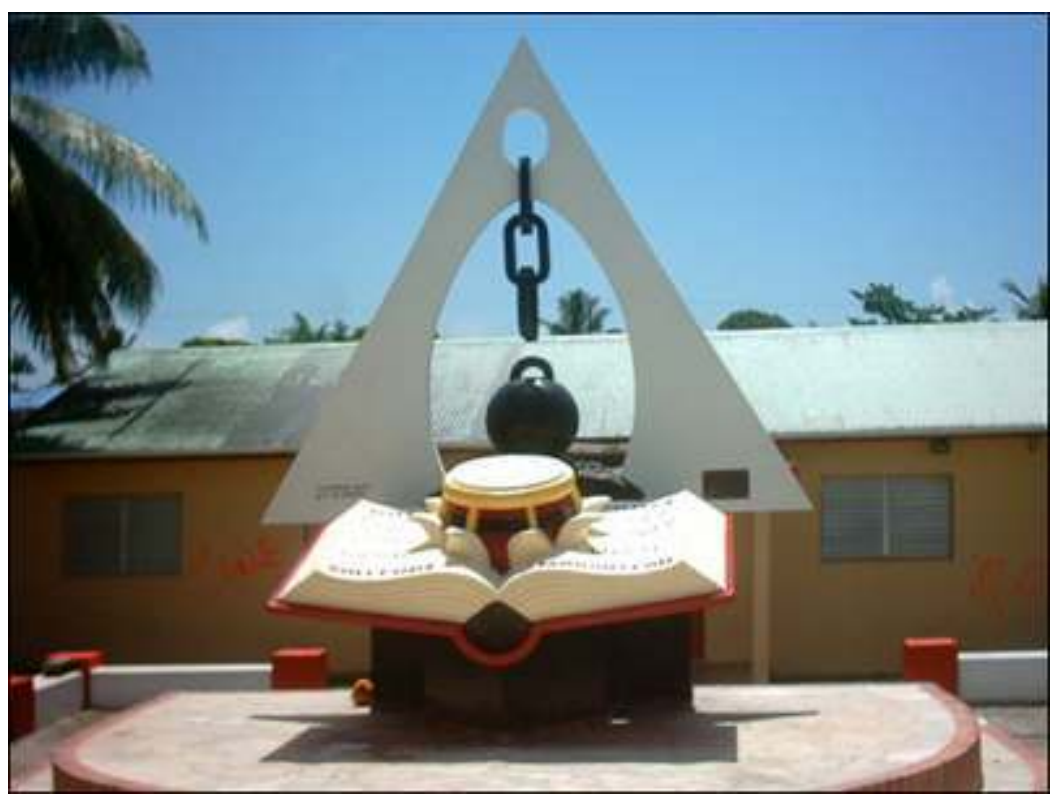

Cliché de l'auteur

18 Une troisième catégorie regroupe les lieux de mémoire qui célèbrent la liberté. On retrouve en effet des lieux de mémoire qui immortalisent la joie et le bonheur des esclaves à l'annonce du décret du 27 avril 1848. Ce sont généralement des fresques qui matérialisent ces sentiments en particulier sur les murs d'enceinte de bâtiments «publics» tels que les écoles, les lycées, les stades,... Toutefois, des monuments commémorent également cette liberté recouvrée comme à Rémire Montjoly, en Guyane, où une sculpture baptisée "Fiiman", signifiant en Bushitongo «Homme libre ", a été inaugurée en juin 2008 lors des festivités du cent soixantième anniversaire de l'abolition de l'esclavage. 


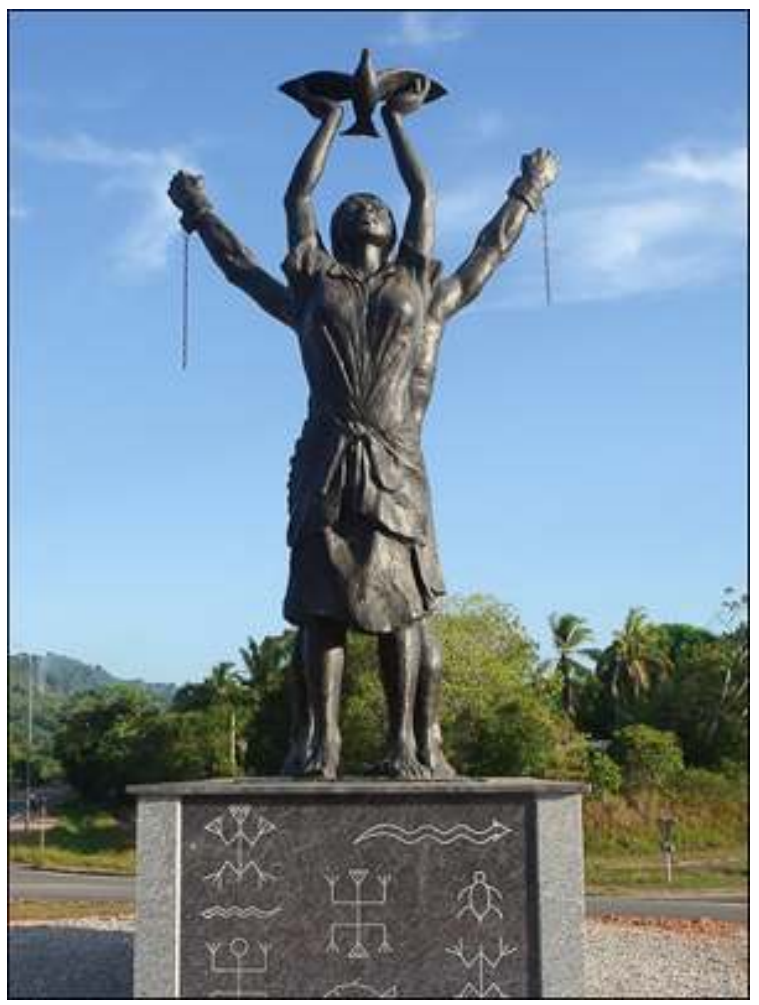

Cliché Isabelle Hidair

19 La quatrième catégorie prend une allure plus solennelle. Les lieux de mémoire qui s'y intègrent s'inspirent en effet des monuments aux morts. Ils rappellent très fortement les cénotaphes qui commémorent les disparus des grandes guerres. L'un des exemples les plus connus est le mémorial des esclaves des Avirons à La Réunion qui reprend l'organisation des monuments aux morts avec une sculpture au premier plan et une liste où s'égrènent les noms d'esclaves réunionnais au second plan. 


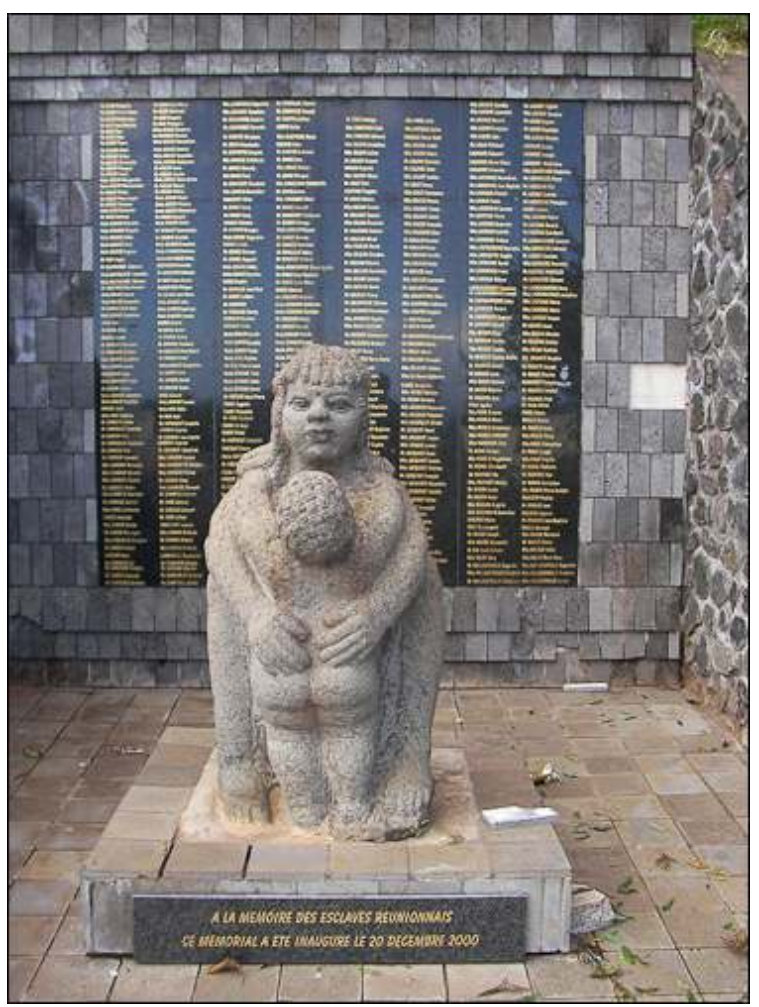

Cliché de l'auteur

la dernière catégorie qui apparaît aujourd'hui comme l'une consacre la résistance à l'esclavage. Un personnage domine tous les autres, celui du rebelle. Il se voit en général doté de deux figures marquantes d'une part celle de l'esclave fugitif qui a échappé à l'asservissement et de l'autre celle de l'esclave insurgé qui a généré des révoltes et mené des séditions.

Dans le premier cas, c'est le nègre marron qui est célébré. Toutefois, cette célébration est assez nuancée suivant les départements. Paradoxalement, elle est la plus significative dans les espaces insulaires où les descendances «marronnes » ne sont pas nettement établies. Il semble clair que si le rapport à l'esclave fugitif en Guyane reste équivoque, il en est tout autrement en Guadeloupe, en Martinique et à La Réunion. Celui-ci n'est plus considéré dans ces îles comme l'expression de l'inhumanité et de la marginalité. Il apparait désormais comme un personnage valeureux qui a su fuir sa condition servile quoiqu'il lui en coûtât. Aux Antilles françaises, l'accent est ainsi mis sur les qualités exceptionnelles de celui qui n'a pas attendu l'abolition de l'esclavage. Les représentations insistent notamment sur sa force physique. La plupart des lieux de mémoire guadeloupéen et martiniquais font figurer en effet un homme robuste et puissant capable de briser ses liens grâce à sa force herculéenne. Les exemples caractéristiques ne manquent pas en particulier dans des communes très touristiques comme Le Diamant en Martinique et Sainte-Anne en Guadeloupe. A la Réunion, cette sensibilité à l'égard du nègre marron s'exprime moins par la force physique que par la reconnaissance de son abnégation. Ce sentiment est particulièrement perceptible dans les hauts de lîle qui ont été marqués par d'illustres «fugitifs » dont l'empreinte se manifeste aujourd'hui encore dans les toponymes (Cimendef, Anchaing, Mafate,...). Ainsi dans la commune de l'Entre- 
deux a été érigée une stèle avec les effigies de marrons légendaires comme le « capitaine » Dimitile et le « roi » Laverdure. Ce lieu de mémoire est par ailleurs devenu au fil des années l'objet d'un nouveau culte. Des associations (Miaro, Capitaine Dimitile et Tradition Salégy) y organisent depuis décembre 2004, la cérémonie "Ati-damba » qui rend hommage aux esclaves malgaches reposant à La Réunion. Cette cérémonie est l'occasion d'une part de manifester le «fihavanana", c'est-à-dire la solidarité et le respect à l'égard de ces prestigieux ancêtres et d'autre part de se recueillir face à ceux qui n'ont pas bénéficié des honneurs funèbres et de sépultures en couvrant notamment la stèle d'un « lamba » (linceul) blanc.

Photo 12 : Le nègre marron à l'entrée du bourg du Diamant (Martinique)

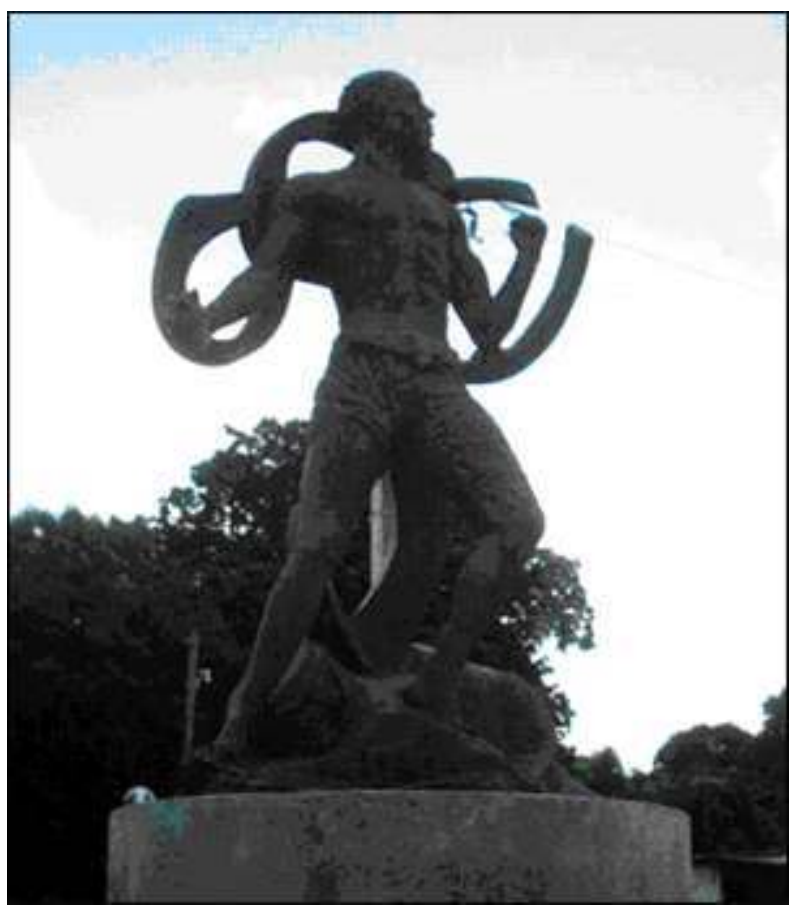

Cliché de l'auteur

Dans le second cas, les lieux de mémoire honorent les esclaves insurgés qui se sont distingués par leur lutte contre le système esclavagiste. Ce genre de lieux se retrouve aux Antilles françaises et de façon notable en Guadeloupe. Cela s'explique par l'existence d'une révolte majeure qui a vu au mois de mai 1802, des groupes de soldats noirs et de couleur émancipés par la première abolition de 1794 s'opposer aux troupes napoléoniennes dirigées par le général Richepance venues rétablir l'esclavage. Cet événement épique, qui accède aujourd'hui au statut de mythe, a véritablement offert la possibilité de célébrer des figures de la résistance et de la bravoure à l'image des officiers Ignace et Delgrès. Des monuments louant leur ténacité ont ainsi été érigés dans l'ensemble de ce département en particulier aux différents ronds-points du boulevard reliant Pointe-à-Pitre aux Abymes. 
Photo 13 - Mémorial de Delgrès et ses compagnons aux Abymes (Guadeloupe)

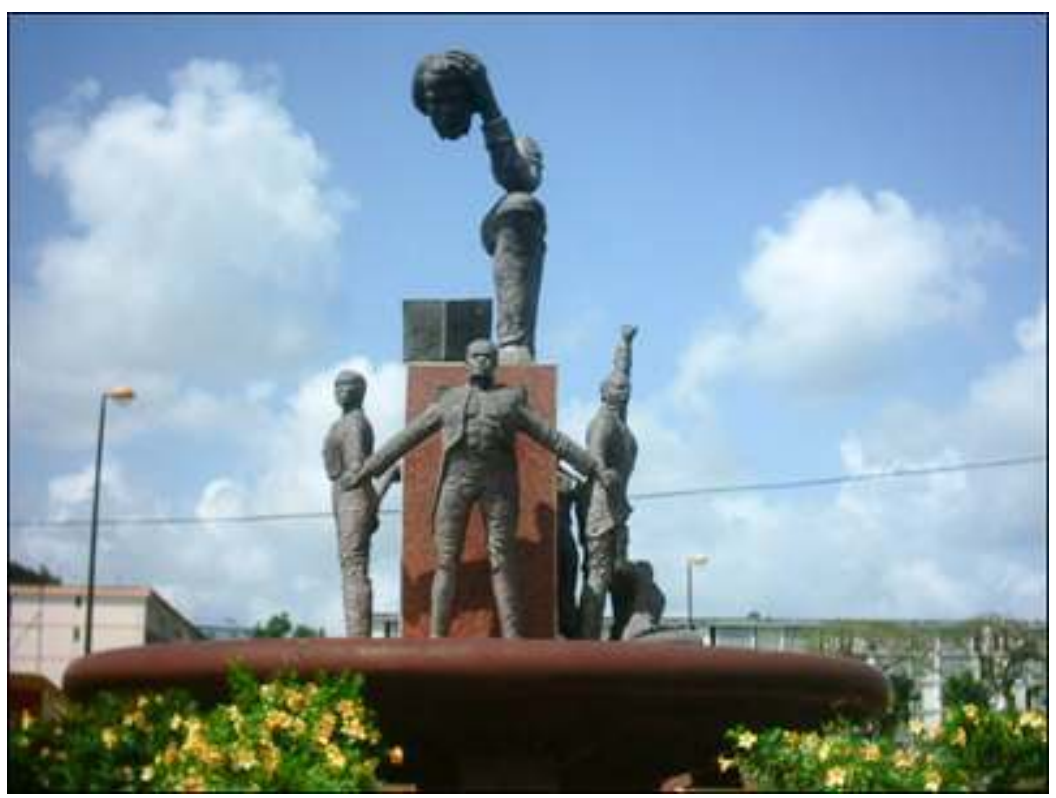

Cliché de l'auteur

Il faut également mentionner la place non négligeable qu'occupent les femmes marronnes et insurgées parmi les figures représentées. Celles qui encourageaient et fanatisaient les hommes de même que celles qui faisaient preuve de bravoure ont aussi leur lieu de mémoire. C'est le cas par exemple à La Réunion de la « Reine » Sarlave, fidèle compagne du « roi » Laverdure, mais aussi de la Mulâtresse solitude ou de la négresse Gertrude qui se distinguèrent en Guadeloupe par leur refus de l'ordre établi en 1802 et 1822 respectivement. 


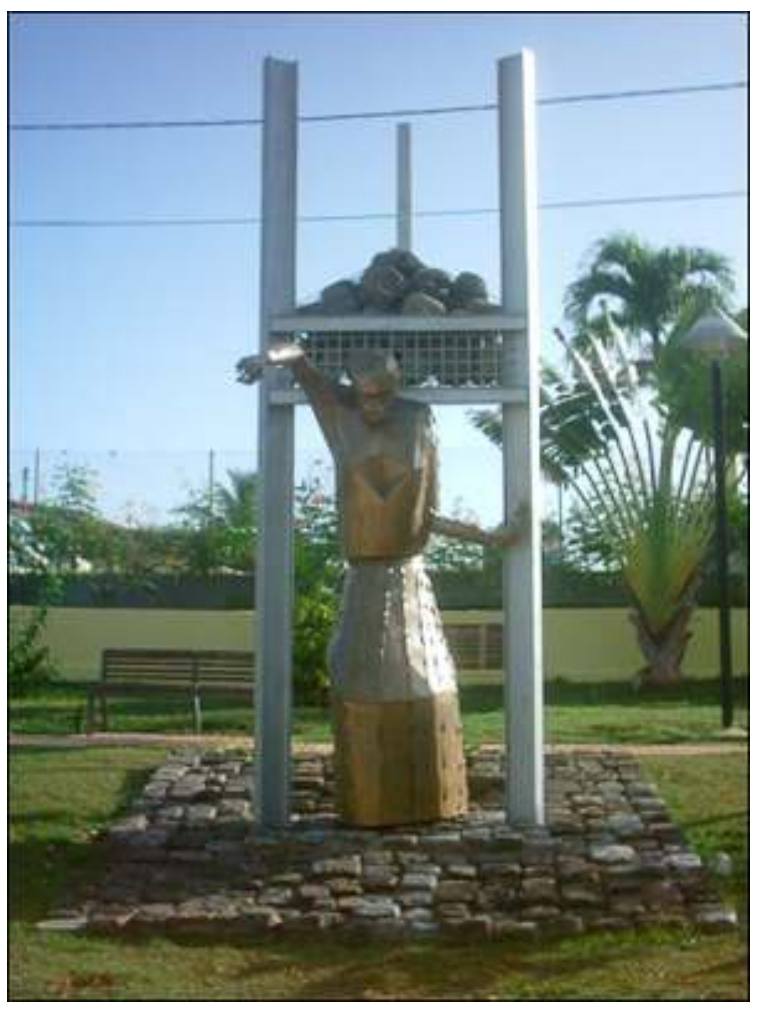

Cliché de l'auteur

24 Au final, on s'aperçoit que les thèmes abordés couvrent un champ qui dépasse la simple problématique abolitionniste à l'origine de ce vaste mouvement d'édification de lieux de mémoire. Ce sont aujourd'hui plus d'une trentaine de marqueurs qui attribuent de nouvelles significations aux territoires ultramarins. Toutefois, ce langage inédit qui se lit dans les paysages ne singularise pas ces départements de leur environnement régional. L'île Maurice, la Barbade, La Jamaïque, Saint-Martin ou le Surinam connaissent la même inflation de lieux de mémoire. L'une des différences réside en réalité dans le fait que cette visibilité ne s'accompagne pas d'une pleine reconnaissance institutionnelle des lieux de mémoire de l'esclavage dans les départements d'outre-mer. A la Réunion par exemple, la question du marronnage ne figurait pas dans le projet de candidature des hauts de l'île au patrimoine mondial de l'humanité alors que dans l'île voisine, Maurice, le projet retenu, celui du morne Brabant, privilégiait au contraire l'histoire dramatique des esclaves en fuite. Il faut dire que la condition d'historicité nécessaire à une consécration institutionnelle dans les départements d'outre-mer ne semble pas assurée.

Tableau 1 - Les thèmes et références symboliques des lieux de mémoire de l'esclavage

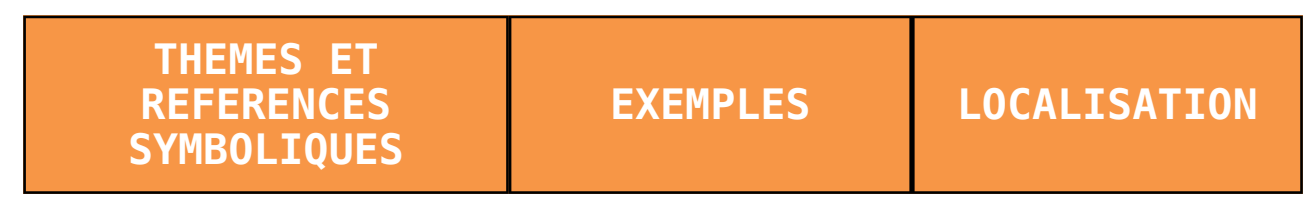




\begin{tabular}{|c|c|c|}
\hline Origine des esclaves & $\begin{array}{c}\text { Mémorial Cap } 110 \\
\text { Statues de la route } \\
\text { de l'esclavage }\end{array}$ & $\begin{array}{c}\text { Diamant, Martinique } \\
\text { Saint-Paul, La } \\
\text { Réunion }\end{array}$ \\
\hline $\begin{array}{l}\text { Les attributs de } \\
\text { L'esclave } \\
\text { (Chaînes, boulets, } \\
\text { tambours,...) }\end{array}$ & $\begin{array}{c}\text { Mémorial de Saint- } \\
\text { François } \\
\text { Mémorial de Saint- } \\
\text { Paul }\end{array}$ & $\begin{array}{l}\text { Saint-François, } \\
\text { Guadeloupe } \\
\text { Saint-Paul, La } \\
\text { Réunion }\end{array}$ \\
\hline $\begin{array}{l}\text { Célébration de la } \\
\text { liberté }\end{array}$ & $\begin{array}{l}\text { La statue de } \\
\text { «Fiiman } »\end{array}$ & $\begin{array}{l}\text { Rémire-Montjoly, } \\
\text { Guyane }\end{array}$ \\
\hline Cénotaphes & $\begin{array}{l}\text { Mémorial des } \\
\text { esclaves réunionnais }\end{array}$ & $\begin{array}{l}\text { Les Avirons, La } \\
\text { Réunion }\end{array}$ \\
\hline $\begin{array}{l}\text { Résistance féminine } \\
\text { l'esclavage }\end{array}$ & $\begin{array}{c}\text { Statue de la } \\
\text { Mulâtresse Solitude } \\
\text { Statue de la } \\
\text { Négresse Gertrude }\end{array}$ & $\begin{array}{l}\text { Pointe-à-Pitre, } \\
\text { Guadeloupe } \\
\text { Petit-Bourg, } \\
\text { Guadeloupe }\end{array}$ \\
\hline $\begin{array}{l}\text { Résistance masculine } \\
\text { l'esclavage }\end{array}$ & $\begin{array}{l}\text { Statue du nègre } \\
\text { marron } \\
\text { Stèle des marrons } \\
\text { Mémorial de Delgrès } \\
\text { et ses compagnons }\end{array}$ & $\begin{array}{c}\text { Diamant, Martinique } \\
\text { Entre-Deux, La } \\
\text { Réunion } \\
\text { Les Abymes, } \\
\text { Guadeloupe }\end{array}$ \\
\hline
\end{tabular}

\section{De la difficile intégration au patrimoine : la condition d'historicité en jeu?}

A la différence des lieux de mémoire anciens, les plus récents ont véritablement du mal à acquérir une dimension historique. Et pourtant une tendance à l'historicisation de la mémoire de l'esclavage semble en cours au niveau national. En premier lieu, on assiste à la multiplication des travaux historiques sur le phénomène et à son intégration progressive dans les manuels scolaires. D'autre part, les questions de l'esclavage et de la traite négrière ont fait l'objet d'une reconnaissance officielle, d'abord par le vote de la loi Taubira par le Parlement en 20017, puis par l'instauration de nouvelles journées de commémoration (le 10 mai et le 23 mai) ${ }^{8}$. A titre d'exemple, la circulaire du 29 avril 2008 du Premier ministre François Fillon indiquait que :

«La journée de commémoration du 10 mai, comme chacune des dates rappelées dans cette circulaire, contribue à l'unité et à la cohésion sociale de notre pays, en permettant notamment aux jeunes générations d'en connaître toute son histoire, dans le partage, en évitant d'occulter, de fragmenter, de limiter le champ chronologique et géographique, voire d'opposer des faits et des mémoires. A ce titre, ces journées participent pleinement au calendrier français de la mémoire ».

Néanmoins, cette évolution est fortement contrariée par un contexte passionné où le débat est loin d'être apaisé. En effet, à un moment où la société française s'interroge sur son identité et son destin collectif ${ }^{9}$, nombreux sont ceux qui voient d'un mauvais œil la 
relecture de l'histoire de la Nation. Les critiques se font ainsi virulentes parmi lesquelles se distinguent trois principales :

- La première critique porte sur la nécessité d'en finir avec la repentance. Selon cette conception, les Français n'ont pas à rouvrir ces pages sombres du passé colonial et à les ressasser, mais se doivent au contraire préserver le récit national. De nombreux intellectuels défendent cette position parmi lesquels des philosophes et des historiens ${ }^{10}$ et parfois même des hommes politiques de premier plan. Ils considèrent que relancer le débat sur l'esclavage pousse à adopter une attitude - que certains n'hésitent pas à qualifier de mal « typiquement français »- qu'il faut combattre. Parmi les conduites à tenir, l'une des plus aptes à s'accommoder au roman national et à lutter contre cette forme de « masochisme » consisterait à rappeler que « l'homme occidental développa l'esclavage plus tardivement (... ), le fit porter sur moins d'Africains que les Arabes (...) et fut de plus le seul promoteur et garant sévère de l'abolitionnisme $»^{11}$ (Raffard de Brienne, 2007).

- Une deuxième critique touche au fait que cette évolution renferme les germes d'une concurrence des mémoires. Ainsi, reconnaître la mémoire de l'esclavage ouvrirait la porte à une surenchère de toute communauté ayant subi un crime atroce et à un enfermement dans une posture victimaire pour lequel il n'y aurait aucune forme d'échappatoire. Le danger serait d'entrer dans un cycle alliant une inflation et un abus de mémoire où chaque communauté serait amenée d'un côté à compter ses morts et à établir une hiérarchie de la douleur et de l'autre à réclamer à terme une stèle, un mémorial, une loi voire des réparations pour le préjudice subi. Le Collectif «Liberté Pour l'Histoire » créé en décembre 2005 par dix-neuf historiens de renom stigmatise notamment la volonté d'exiger la reconnaissance publique des torts subis en prenant comme exemple notable le risque que le génocide des Vendéens de 1793-1794 mené sous la Convention soit un jour reconnu par le législateur et fasse par la suite l'objet de revendications particulières ${ }^{12}$.

- Une troisième critique fait miroiter le spectre du communautarisme. Selon les tenants de cette vision, plus le passé esclavagiste de la France est évoqué et plus grande est la propension des communautés à se refermer sur elles-mêmes. On remarque que dans une large mesure, les émeutes urbaines de novembre 2005 ont renforcé cette crainte. Elles ont alimenté l'idée que des jeunes issus de l'Outre-mer ou de l'immigration vivant dans les banlieues des grandes villes y chercheraient des raisons à des processus de ségrégation et de relégation socio-spatiales. Aussi pour beaucoup, la République française, une et indivisible, n'a pas vocation à encourager une dynamique mémorielle soumise au bricolage et à la manipulation qui risquerait de mettre à mal à terme la cohésion de la société toute entière.

Au total, la tendance à l'historicisation des lieux de mémoire de l'esclavage qui ont émergé dans les DOM depuis la fin du siècle dernier se heurte à de vrais obstacles. L'une des manifestations les plus significatives de ces difficultés est la multiplication de pétitions et de rapports visant la loi reconnaissant l'esclavage comme crime contre l'humanité et les journées de commémoration. Le rapport de la commission de réflexion sur la modernisation des commémorations publiques remis par l'historien A. Kaspi en novembre 2008 au Secrétaire d'Etat à la Défense et aux Anciens combattants en témoigne largement. Rappelant qu'il «n'est pas admissible que la nation cède aux intérêts communautaristes et que l'on multiplie les journées de repentance pour satisfaire un groupe de victimes » (Kaspi, 2008), il préconise de revenir sur l'instauration de journées de commémoration de l'esclavage et des traites. Dans ce contexte, il semble vraisemblable que la pleine intégration des lieux de mémoire de l'esclavage au patrimoine soit amenée à 
passer par des stratégies de négociation entre cette mémoire singulière et le récit national.

\section{BIBLIOGRAPHIE}

Adelaïde-Merlande Jacques, Delgrès ou la Guadeloupe en 1802, Karthala, Paris, 1986.

Bancel Nicolas et al., (dir.), Culture post-coloniale, 1961-2006, Traces et mémoires coloniales en France, Paris, Editions Autrement, 2006.

Bangou Henri, La Révolution et l'esclavage à la Guadeloupe, 1789-1802, L'Harmattan, Paris, 1990.

Bessière Philippe, Vingt Décembre : le jour où La réunion se souvient..., L'Harmattan, Paris, 2001.

Bonniol Jean-Luc, « De la construction d'une mémoire historique aux figurations de la traite et de l'esclavage dans l'espace public antillais » in Bonniol Jean-Luc et Crivello Maryline (dir.), Façonner le passé. Représentations et cultures de l'histoire (XVI ${ }^{e}-\mathrm{XXI}^{e}$ siècle), Aix-en-Provence : Presses de l'Université de Provence, 2004.

Brown Laurence, «Creole Bonapartism on Post-Emancipation Society : Martinique's Monument to the Empress Joséphine ", Revue de la Société Française d'Histoire d'Outre-mer, 183, 2006, $1^{\mathrm{er}}$ semestre.

Chivallon Christine, « Mémoires antillaises de l'esclavage », Ethnologie française, XXXII (4), 2002.

Chivallon Christine, « Rendre visible l'esclavage aux Antilles françaises (Martinique) : muséographie et lieux de mémoire ou les hiatus d'une mémoire difficile à énoncer ", L'homme, $180,2006$.

Collectif. Le patrimoine des communes de la Guadeloupe, de la Martinique, de la Guyane et de La Réunion, Paris, Editions Le Flohic, 4 volumes, 1998-2000.

Cottias Myriam, «L'oubli du passé contre la citoyenneté : troc et ressentiment à la Martinique (1848-1946) », in Constant F., Daniel J. (dir), 1946-1996 : Cinquante ans de départementalisation outremer, Paris : L'Harmattan, 1997.

Cottias Myriam, « La politique de l'oubli », in « Cent cinquantenaire de l'abolition de l'esclavage », France-Antilles, Hors-Série, mai 1998.

Dorigny Marcel, (dir.), 1999, Esclavage, résistances et abolition, Editions du CTHS, Paris.

Dubois Laurent, 1998, Les Esclaves de la République, l'histoire de la première abolition, 1789-1794, Calmann-Lévy, Paris

Dubois Laurent, "Solitude's Statue : Confronting the Past in the French Caribbean ", Revue de la Société Française d'Histoire d'Outre-mer, 183, 2006, $1^{\mathrm{er}}$ semestre.

Eve Prosper, Le 20 décembre 2004 : Le temps de la parole, Océan Editions, Saint-André, 2005.

Fuma Sudel, L'esclavagisme à la Réunion, 1794-1848, L'harmattan/ Université de La Réunion, Paris, 1992. 
Gautier Arlette, Les Sours Solitude. La condition féminine dans l'esclavage aux Antilles du XVII au XIX siècle, Etudes Caribéennes, Paris, 1985.

Giordani Jean-Pierre. La Guadeloupe face à son patrimoine, Itinéraires et modalités d'une reconnaissance et d'une revalorisation, Paris : Karthala, 1996.

Girault Arthur, Des Rapports entre métropole et colonies, Institut Colonial International, Bruxelles, 1903.

Hidair Isabelle, La construction identitaire des Créoles de Guyane. De la gestion d'un héritage pluriel à l'insertion dans un contexte multiculturel, Thèse de doctorat d'anthropologie, EHESS-CEAF, Paris, 2003.

Jauze Jean-Michel, (2000), Villes et patrimoine à La Réunion, Paris, L’Harmattan,

Kaspi André, (dir.), Rapport de la commission de réflexion sur la modernisation des commémorations publiques, Secrétariat d'Etat à la Défense et aux Anciens combattants, Paris, 2008,

Keslassy Eric, Rosembaum Alexis, Mémoires vives, Paris : Bourin Editeur, 2007

Léonard Yves, (dir.), « La mémoire, entre histoire et politique », Les Cahiers français, Paris : La documentation française, Juillet-août 2001.

Mam Lam Fouck Serge, L'esclavage en Guyane entre l'occultation et la revendication. L'évolution de la représentation de l'esclavage dans la société guyanaise (1848-1977), Presses Universitaires Créoles/ Gerec, 1998.

Nicolas Thierry, «Les Antilles françaises entre insularité et continentalité : un essai sur la logique d'intégration de la Guadeloupe et de la Martinique à l'espace national et européen ", Thèse de doctorat de Géographie, Université Paris-Sorbonne, 2002.

Nicolas Thierry, «Politique patrimoniale et patrimonialisation aux Antilles françaises », Techniques et cultures, Revue d'anthropologie des techniques, MSH-CNRS, Paris, Décembre 2003.

Nora Pierre, (dir.), Les lieux de mémoire, Bibliothèque illustrée des histoires, 3 volumes, Paris, Gallimard, 1984-1992.

Poirier Jean, Fuma Sudel, «La mémoire de l'esclave. Survivances et permanences » in Fuma Sudel (dir.), Mémoire orale et esclavage dans îles du sud-ouest de l'Océan indien: Silences, oublis, reconnaissance, Actes du colloque international de Saint-Denis, Saint-Denis : Unesco-Université de la Réunion, 2004.

Raffard de Brienne Arnaud, La désinformation autour de l'esclavage, Paris : L'étoile du Berger, 2006 Ricoeur Paul, La mémoire, l'histoire, l'oubli, Paris : Le Seuil, 2000.

Riegl Alois, Le culte moderne du monument, son essence, sa genèse, Paris : Editions du Seuil, Traduction de la version allemande (1903), 1984.

Todorov Tzvetan, Mémoire du mal, intention du bien, Paris, Editions Robert Laffont, 2000.

Vergès Françoise, « Mémoires visuelles et virtuelles à l'île de La Réunion », Cahier d'études africaines, Editions de l'EHESS-CNRS, Paris, 2004.

Vergès Françoise, La mémoire enchaînée. Questions sur l'esclavage, Albin, Michel Paris, 2006. 


\section{NOTES}

1. Sous l'appellation de lieux de mémoire, je regroupe des traces matérielles qui se voient investies du sens résultant de la quête mémorielle d'un groupe humain. Il faut donc entendre ici par lieux de mémoire de l'esclavage des monuments, des statues, des stèles, des plaques commémoratives, des sculptures ou encore des fresques liés à cette période qui s'est étendue dans l'outre-mer français du XVII ${ }^{\mathrm{e}}$ siècle au milieu du XIX ${ }^{\mathrm{e}}$ siècle.

2. Cette statue dont la création fut soutenue financièrement par Napoléon III est apparue à l'époque comme le symbole d'un Bonapartisme créole. D'ailleurs sur le piédestal de cette œuvre exécutée par Vital Dubray figure l'inscription suivante: "L'an MDCCCLVIII/ Napoléon III régnant/ Les habitants de la Martinique/ Ont élevé ce monument ». Voir à ce sujet l'article de Laurence Brown, «Creole Bonapartism on Post-Emancipation Society : Martinique's Monument to the Empress Joséphine", Revue de la Société Française d'Histoire d'Outre-mer, 183, 2006, $1^{\mathrm{er}}$ semestre. Il faut par ailleurs noter que cette statue a été décapité puis maculée de peinture rouge au début des années 1990.

3. Voir à ce sujet les travaux de Myriam Cottias.

4. Loi $n^{\circ} 83-550$ du 30 juin 1983 relative à la commémoration de l'abolition de l'esclavage: "Article unique : La commémoration de l'abolition de l'esclavage par la République française et de la fin de tous les contrats d'engagement souscrits à la suite de cette abolition font l'objet d'une journée fériée dans les départements de la Guadeloupe, de Guyane, de la Martinique, de La Réunion ainsi que dans la collectivité territoriale de Mayotte. Un décret fixe la date de cette commémoration pour chacune des collectivités visées ci-dessus et précise les conditions dans lesquelles cette commémoration sera célébrée sur le territoire métropolitain ». Les dates fixées par décret pour les quatre DOM sont le 22 mai pour la Martinique, le 27 mai pour la Guadeloupe, le 10 juin pour la Guyane et le 20 décembre pour La Réunion.

5. Cf. Girault A., 1903, Des Rapports entre métropole et colonies, Institut Colonial International, Bruxelles.

6. En Guadeloupe principalement et en Martinique dans une moindre mesure de nouvelles organisations indépendantistes décidèrent au tournant des années 1970-1980 de privilégier l'action directe plutôt que l'action démocratique en posant la question du particularisme antillais et de la souveraineté nationale en terme guérillériste. Le Groupe de Libération Armé (GLA), issu du Mouvement Pour une Guadeloupe Indépendante (MPGI), fut le premier à prendre la voie des armes s'attaquant aux intérêts de l'Etat et aux individus. Ainsi en mars 1980, il s'attribue la tentative d'assassinat de Raymond Viviès, conseiller général de la Guadeloupe et vigoureusement anti-indépendantiste.Puis c'est au tour de l'Alliance Révolutionnaire Caraïbe (ARC) de prendre les armes en 1983 et de revendiquer de nouveaux attentats touchant en Guadeloupe comme en Martinique, des bâtiments publics tels que les palais de justice, les casernes de gendarmerie, le siège de la télévision publique.

7. Cf. la Loi $n^{\circ} 2001-434$ du 21 mai 2001 tendant à la reconnaissance de la traite et de l'esclavage en tant que crime contre l'humanité.

8. Si le 10 mai, jour des «mémoires de la traite, de l'esclavage et de leurs abolitions », reste la date la plus connue et célébrée dans l'hexagone, la circulaire du 29 avril 2008 du Premier ministre François Fillon a instauré une seconde date de commémoration le 23 mai : «La date du 23 mai sera, pour les associations regroupant les Français d'outre-mer de l'Hexagone, celle de la commémoration du passé douloureux de leurs aïeux qui ne doit pas être oublié. (...) Cette date rappelle, d'une part, celle de l'abolition de l'esclavage en 1848 et, d'autre part, celle de la marche silencieuse du 23 mai 1998 qui a contribué au débat national aboutissant au vote de la loi reconnaissant l'esclavage comme un crime contre l'humanité. (...)». 
9. L'apparition du terme «identité nationale» dans l'appellation d'un ministère en 2007 témoigne de cette interrogation: «Le ministère l'immigration, de l'intégration, de l'identité nationale et du codéveloppement ».

10. Voir entre autres P. Bruckner (La tyrannie de la pénitence. Essai sur le masochisme occidental), D. Lefeuvre (Pour en finir avec la repentance), J.-P. Rioux (La France perd la mémoire. Comment un pays démissionne de son histoire) ou M. Gallo (fier d'être français)

11. A. Raffard de Brienne précise ainsi que «les Arabo-musulmans et les Africains eux-mêmes, avec leurs traites internes furent si l'on considère les données les plus récentes, sur l'aspect quantitatif, les plus grands négriers de l'histoire de l'humanité ». Cet auteur s'appuie notamment sur l'argumentaire développé par l'historien O. Pétré Grenouilleau et que défendent également des géographes comme Y. Lacoste (Voir les numéros de la revue Hérodote notamment le $\mathrm{n}^{\circ}$ 117).

12. Voir l'article introductif du site internet de l'association Liberté Pour l'Histoire (LPH) : http://www.lph-asso.fr/ Les 19 signataires sont: Jean-Pierre Azéma, Elisabeth Badinter, JeanJacques Becker, Françoise Chandernagor, Alain Decaux, Marc Ferro, Jacques Julliard, Jean Leclant, Pierre Milza, Pierre Nora, Mona Ozouf, Jean-Claude Perrot, Antoine Prost, René Rémond, Maurice Vaïsse, Jean-Pierre Vernant, Paul Veyne, Pierre Vidal-Naquet et Michel Winock.

\section{RÉSUMÉS}

Parmi les phénomènes communs aux départements d'outre-mer, le processus d'élaboration de repères mémoriels liés à l'esclavage est certainement l'un des plus significatifs de la dernière décennie. De l'Océan Atlantique à l'Océan Indien, il existe en effet le même souci de porter un regard différent sur la période esclavagiste et d'inscrire des lieux de mémoire dans les paysages. Néanmoins, ces lieux pourvus d'un sens nouveau peinent à accéder au statut d'éléments du patrimoine. De façon paradoxale, les obstacles à leur patrimonialisation ne tiennent pas à l'idée que de tels lieux pourraient constituer des symptômes de la résurgence d'une idéologie nationaliste mais résident plutôt dans leur déficit d'historicité.

Among the phenomena common to French overseas departments, the reproduction of memorial markers associated with slavery is certainly one of the most significant of the last decade. From the Atlantic Ocean to the Indian Ocean, there is the same desire to introduce a different perspective on the slave period and to include commemorative sites in the environment. However, these memory places can hardly obtain the status of heritage components. In a paradoxical way, the obstacles to their integration in the Heritage are not related to the symptoms of the resurgence of a secessionist ideology. In this paper we will prove that the real cause of this non-integration lies to the lack of historicity of such places.

\section{INDEX}

Keywords : Memory places, Slavery, Heritage, Guadeloupe, Martinique, French Guiana, Reunion Island.

Mots-clés : Lieux de mémoire, Esclavage, Patrimoine, Guadeloupe, Martinique, Guyane, Réunion 


\section{AUTEUR}

\section{THIERRY NICOLAS}

Thierry Nicolas (thierrynicolas@wanadoo.fr) est enseignant au Département de Géographie de l'Université de La Réunion et chercheur associé au CREGUR et à l'UMR PRODIG 